\title{
Best candidate routing algorithms integrated with minimum processing time and low blocking probability for modern parallel computing systems
}

\author{
Aadel M. Alatwi ${ }^{1}$, Ahmed Nabih Zaki Rashed ${ }^{2}$, Ahmed M. El-Eraki ${ }^{3}$, IS Amiri ${ }^{4}$ \\ ${ }^{1}$ Electrical Engineering Department, Faculty of Engineering, Tabuk University, Saudi Arabia \\ ${ }^{2}$ Electronics and Electrical Communications Engineering Department, Faculty of Electronic Engineering, \\ Menoufia University, Egypt \\ ${ }^{3}$ Faculty of Engineering, Telecommunication Department, Egyptian Russian University, Egypt \\ ${ }^{4}$ Computational Optics Research Group, Advanced Institute of Materials Science, Ton Duc Thang University, \\ Ho Chi Minh City, Vietnam \\ ${ }^{4}$ Faculty of Applied Sciences, Ton Duc Thang University, Ho Chi Minh City, Vietnam
}

\begin{tabular}{l} 
Article Info \\
\hline Article history: \\
Received Jan 10, 2020 \\
Revised Mar 11, 2020 \\
Accepted Mar 25, 2020 \\
\hline
\end{tabular}

\section{Keywords:}

Blocking probability

Network delay time

Processing time

Routing algorithms

\begin{abstract}
This study has clarified the best candidate routing algorithms integrated with minimum processing times and low blocking probabilities for modern parallel computing systems. Different methods were employed, such as the fast window method (FWM), fast bitwise window method (FBWM), and fast improved window method (FIWM), to upgrade the processing time and reduce the network delay time. In addition, different algorithms were studied such as the fast window ascending, the fast window descending, the fast window sequential algorithm, and the fast window sequential down algorithms; these were studied to show the numerical results of the networks' blocking probabilities, processing times, and delay times.
\end{abstract}

Copyright $(0) 2020$ Institute of Advanced Engineering and Science. All rights reserved.

\section{Corresponding Author:}

IS Amiri,

Ton Duc Thang University,

Ho Chi Minh City, Vietnam.

E-mail: irajsadeghamiri@tdtu.edu.vn

\section{RELATED WORKS}

Optical communication networks for optical computing systems have had an important role in researching optics, once the fiber optic bandwidth capacity advantages of the optical domain became evident [1-5]. Supported by the previous research in the fiber optic field of high-speed optical communication and computing networks, high-performance parallel computing systems could achieve low network latencies [6-10], low processing times, and high throughput or transmission data rates of interconnectivity between processing elements [11-15]. The basic requirements of the new high fiber optic bandwidth applications, such as medical imaging, video services [16-18], and distributed central processing unit interconnections, require basic recent or modern solutions with high transmission bit rates or throughputs. These solutions emulate the needs of the recent fiber optic communication systems which reach the limits of terabits per second for achieving high-speed performance for optical computing networks with minimum blocking probabilities [19-25].

\section{MODEL DESCRIPTION AND RESEARCH METHOD}

There are many types of routing: fixed routing, adaptive routing, and alternate routing. The basic advantage of fixed routing is the lack of a central processing unit for route selection [20]. The main drawback 
of fixed routing is the increased blocking probability because of its convergence. The main advantage of the adaptive algorithm is to avoid a highly-loaded route, but its main drawback is the necessity for the link-state information exchange [21-24]. The basic advantage of alternate algorithms is to carry retry other routes if the establishment fails, but its main drawback are results in the wrong route selection [26-31].

We have studied the fast window method (FWM), fast bitwise window method (FBWM), and fast improved window method (FIWM). In addition to the fast window ascending algorithm, the fast window descending, fast window sequential, and fast window sequential down algorithms were are also studied. All of the proposed fast window methods and routing algorithms are clarified in the numerical results to show the network processing time, network delay time, and final network blocking probability. All of the numerical results are compared with the previous conventional window methods and traditional routing algorithms.

\section{PERFORMANCE ANALYSIS WITH DISCUSSIONS}

Figure 1 presents the processing time and network delay time variations against the network size variations for the previous window method, and it proposes a fast window method. The processing time for the traditional window method is $0.35 \mathrm{~ms}$, and the processing time for the proposed fast WM is $0.09 \mathrm{~ms}$ for the small network size value of 8 . The processing time for the traditional window method is $18.13 \mathrm{~ms}$, and the processing time for the proposed fast $\mathrm{WM}$ is $1.2 \mathrm{~ms}$ for the medium network size value of 128 . The processing time for the traditional window method is $1392 \mathrm{~ms}$, and the processing time for the proposed fast WM is $148.5 \mathrm{~ms}$ for the large network size value of 1024. In the same way, the network delay time for the traditional window method is $2.5 \mathrm{~ms}$, and the network delay time for the proposed fast WM is $1.5 \mathrm{~ms}$ for the small network size value of 8 . However, the network delay time for the traditional window method is 40 $\mathrm{ms}$, and the network delay time for the proposed fast WM is $24 \mathrm{~ms}$ for the medium network size value of 128. The network delay time for the traditional window method is $320 \mathrm{~ms}$, and the processing time for the proposed fast WM is $188 \mathrm{~ms}$ for the large network size value of 1024 .

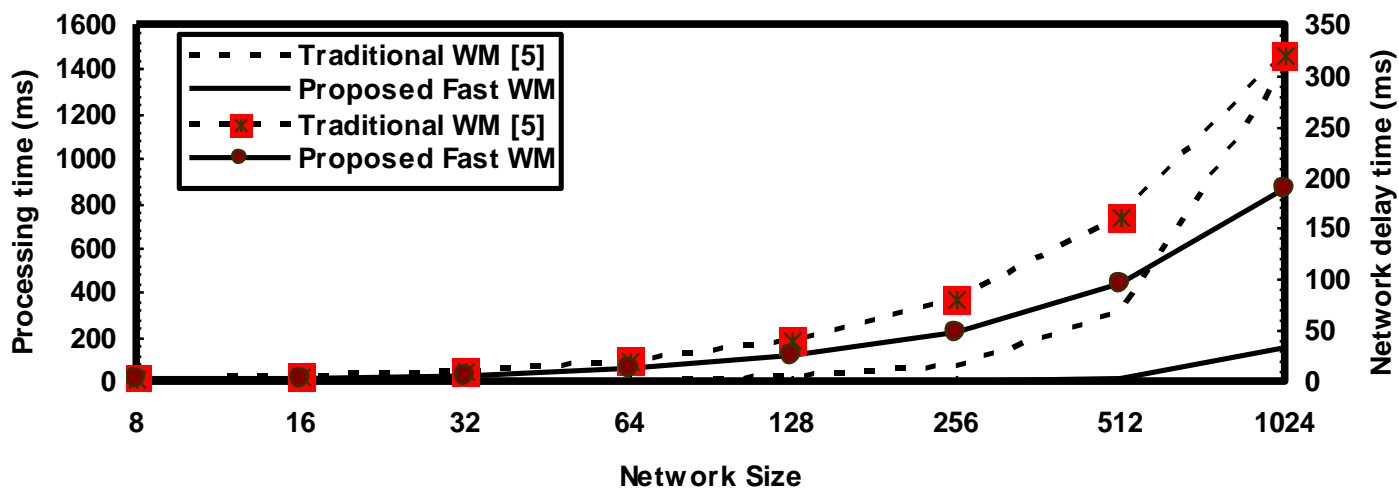

Figure 1. Processing time and network delay time variations against network size variations for the previous window method and the proposed fast window method

Figure 2 indicates the relation between the processing time and network delay time variations against network size variations for the previous improved window method and the proposed fast, improved window method. The processing time for the traditional improved window method is $0.18 \mathrm{~ms}$, and the processing time for the proposed fast improved WM is $0.03 \mathrm{~ms}$ for the small network size value of 8 . The processing time for the traditional improved window method is $30.35 \mathrm{~ms}$, and the processing time for the proposed fast improved WM is $1.87 \mathrm{~ms}$ for the medium network size value of 128. The processing time for the traditional improved window method is $1228 \mathrm{~ms}$, and the processing time for the proposed fast improved WM is $150 \mathrm{~ms}$ for the large network size value of 1024. In the same way, the network delay time for the traditional improved window method is $5 \mathrm{~ms}$, and the network delay time for the proposed fast improved WM is $3 \mathrm{~ms}$ for the small network size value of 8 . However, the network delay time for the traditional improved window method is $80 \mathrm{~ms}$, and the network delay time for the proposed fast improved WM is $48 \mathrm{~ms}$ for the medium network size value of 128. The network delay time for the traditional improved window method is $500 \mathrm{~ms}$, and the processing time for the proposed fast improved WM is $327 \mathrm{~ms}$ for the large network size value of 1024 . 
Figure 3 shows the relation between the processing time and network delay time variations against network size variations for the previous bitwise window method and the proposed fast bitwise window method. The processing time for the traditional bitwise window method is $0.28 \mathrm{~ms}$, and the processing time for the proposed fast bitwise WM is $0.15 \mathrm{~ms}$ for the small network size value of 8 . The processing time for the traditional bitwise window method is $50.35 \mathrm{~ms}$, and the processing time for the proposed fast bitwise $\mathrm{WM}$ is $30 \mathrm{~ms}$ for the medium network size value of 128 .

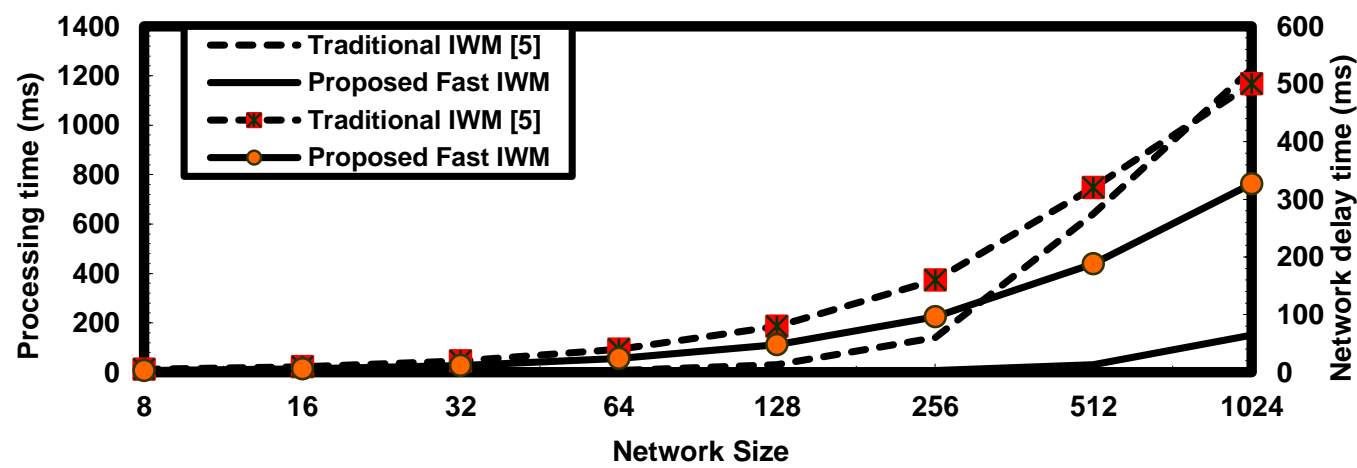

Figure 2. Processing time and network delay time variations against network size variations for the previous improved window method and the proposed fast, improved window method

The processing time for the traditional bitwise window method is $1564 \mathrm{~ms}$, and the processing time for the proposed fast bitwise WM is $657 \mathrm{~ms}$ for the large network size value of 1024. In the same way, the network delay time for traditional bitwise window method is $0.36 \mathrm{~ms}$, and the network delay time for the proposed fast bitwise WM is $0.1 \mathrm{~ms}$ for the small network size value of 8 . The network delay time for the traditional bitwise window method is $5.65 \mathrm{~ms}$, and the network delay time for the proposed fast bitwise WM is $1.6 \mathrm{~ms}$ for the medium network size value of 128 . The network delay time for the traditional bitwise window method is $40.65 \mathrm{~ms}$, and the processing time for the proposed fast bitwise WM is $13 \mathrm{~ms}$ for the large network size value of 1024 .

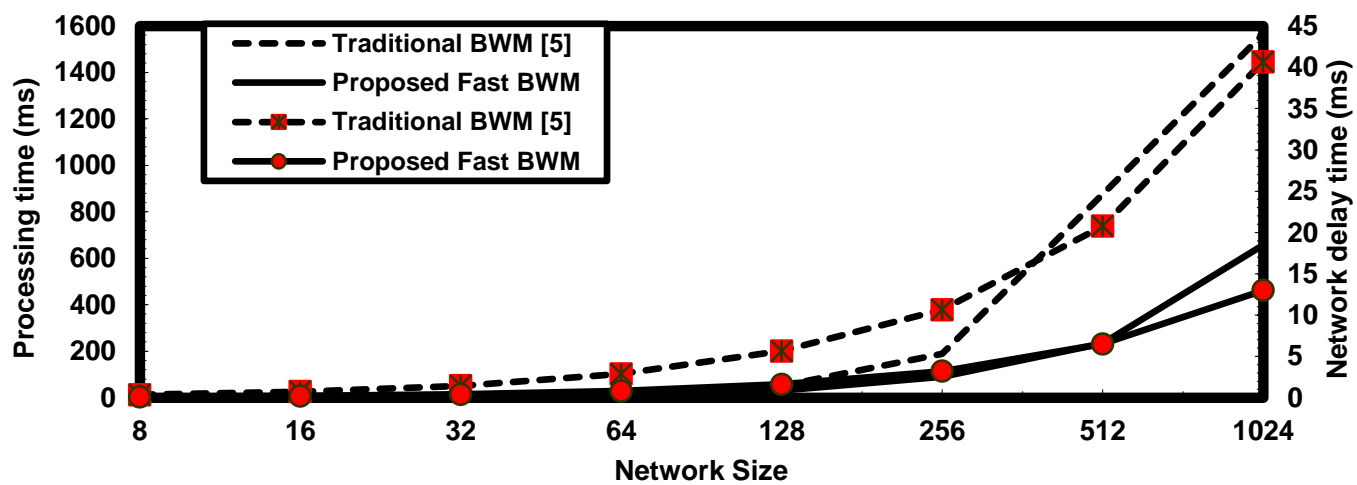

Figure 3. Processing time and network delay time variations against network size variations for the previous bitwise window method and the proposed fast bitwise window method

Figure 4 illustrates the relation between the processing time and network delay time variations against the network size variations for the previous Ascending algorithm and the proposed fast ascending window method. The processing time for the Ascending algorithm is $0.013 \mathrm{~ms}$, and the processing time for the proposed fast Ascending WM is $0.0052 \mathrm{~ms}$ for the small network size value of 8 . The processing time for the Ascending algorithm is $1.742 \mathrm{~ms}$, and the processing time for the proposed fast Ascending WM is 0.217 $\mathrm{ms}$ for the medium network size value of 128. The processing time for the Ascending algorithm is $4.753 \mathrm{~ms}$, and the processing time for the proposed fast Ascending WM is $0.319 \mathrm{~ms}$ for the large network size value of 
1024. In the same way, the network delay time for the Ascending algorithm is $0.2 \mathrm{~ms}$, and the network delay time for the proposed fast Ascending WM is $0.05 \mathrm{~ms}$ for the small network size value of 8 . However, the network delay time for the Ascending algorithm is $3.2 \mathrm{~ms}$, and the network delay time for the proposed fast Ascending WM is $0.8 \mathrm{~ms}$ for the medium network size value of 128 . The network delay time for the Ascending algorithm is $25 \mathrm{~ms}$, and the processing time for the proposed fast Ascending WM is $6.65 \mathrm{~ms}$ for the large network size value of 1024 .

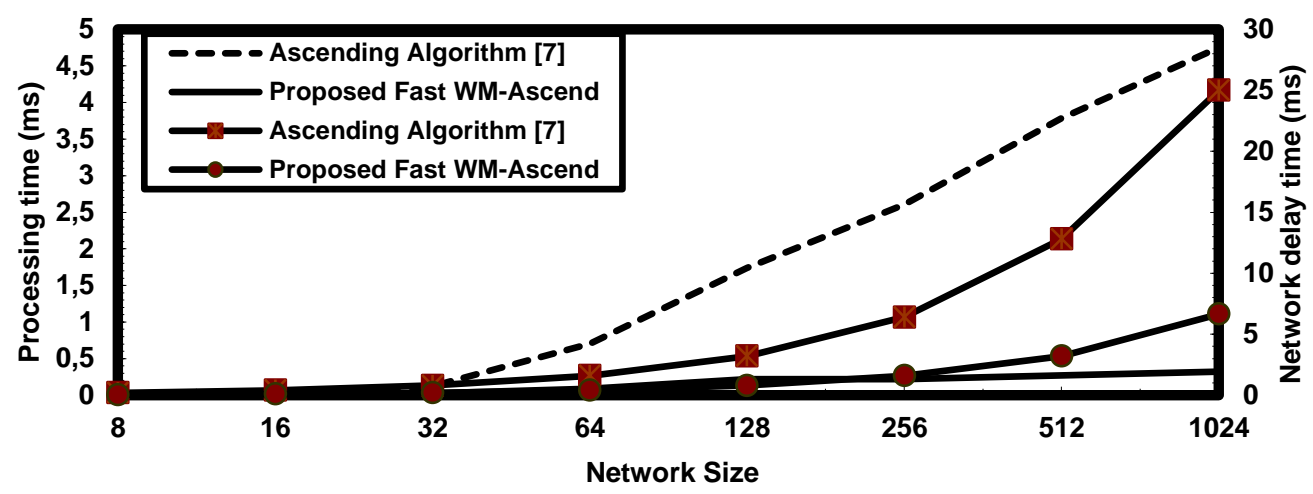

Figure 4. Processing time and network delay time variations against network size variations for the previous Ascending algorithm and the proposed fast ascending window method

Figure 5 clarifies the relation between the processing time and network delay time variations against the network size variations for the previous descending algorithm and the proposed fast descending window method. The processing time for the descending algorithm is $0.016 \mathrm{~ms}$, and the processing time for the proposed fast descending WM is $0.0049 \mathrm{~ms}$ for the small network size value of 8 . The processing time for the descending algorithm is $0.6715 \mathrm{~ms}$, and the processing time for the proposed fast descending WM is 0.145 $\mathrm{ms}$ for the medium network size value of 128 . The processing time for the descending algorithm is $1.243 \mathrm{~ms}$, and the processing time for the proposed fast descending WM is $0.342 \mathrm{~ms}$ for the large network size value of 1024. In the same way, the network delay time for the descending algorithm is $0.031 \mathrm{~ms}$, and the network delay time for the proposed fast descending WM is $0.027 \mathrm{~ms}$ for the small network size value of 8 . The network delay time for the descending algorithm is $0.862 \mathrm{~ms}$, and the network delay time for the proposed fast descending WM is $0.431 \mathrm{~ms}$ for the medium network size value of 128 . The network delay time for the descending algorithm is $1.51 \mathrm{~ms}$, and the processing time for the proposed fast descending WM is $0.746 \mathrm{~ms}$ for the large network size value of 1024 .

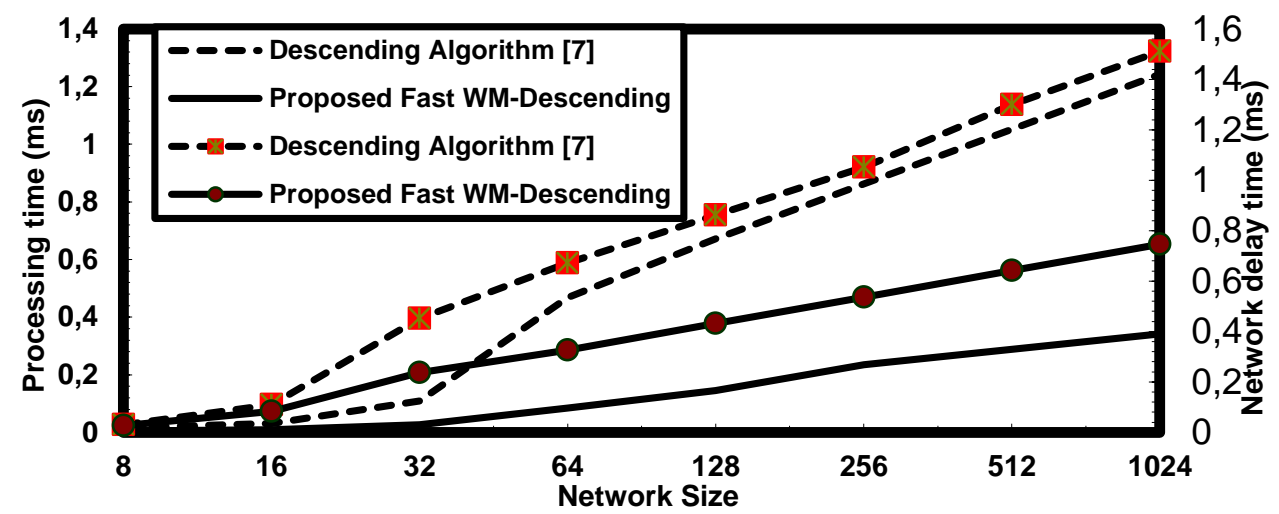

Figure 5. Processing time and network delay time variations against network size variations for the previous descending algorithm and the proposed fast descending window method 
Figure 6 shows the relation between processing time and network delay time variations against the network size variations for the previous sequential algorithm and the proposed fast sequential window method. The processing time for the sequential algorithm is $0.01 \mathrm{~ms}$, and the processing time for the proposed fast sequential WM is $0.005 \mathrm{~ms}$ for the small network size value of 8 . The processing time for the sequential algorithm is $1.425 \mathrm{~ms}$, and the processing time for the proposed fast sequential WM is $0.225 \mathrm{~ms}$ for the medium network size value of 128. The processing time for the sequential algorithm is $3.625 \mathrm{~ms}$, and the processing time for the proposed fast sequential WM is $0.328 \mathrm{~ms}$ for the large network size value of 1024. In the same way, the network delay time for the sequential algorithm is $0.125 \mathrm{~ms}$, and the network delay time for the proposed fast sequential WM is $0.085 \mathrm{~ms}$ for the small network size value of 8 . The network delay time for sequential algorithm is $3.71 \mathrm{~ms}$, and the network delay time for the proposed fast sequential WM is $0.387 \mathrm{~ms}$ for the medium network size value of 128 . The network delay time for sequential algorithm is $6.413 \mathrm{~ms}$, and the processing time for the proposed fast sequential WM is $0.595 \mathrm{~ms}$ for the large network size value of 1024 .

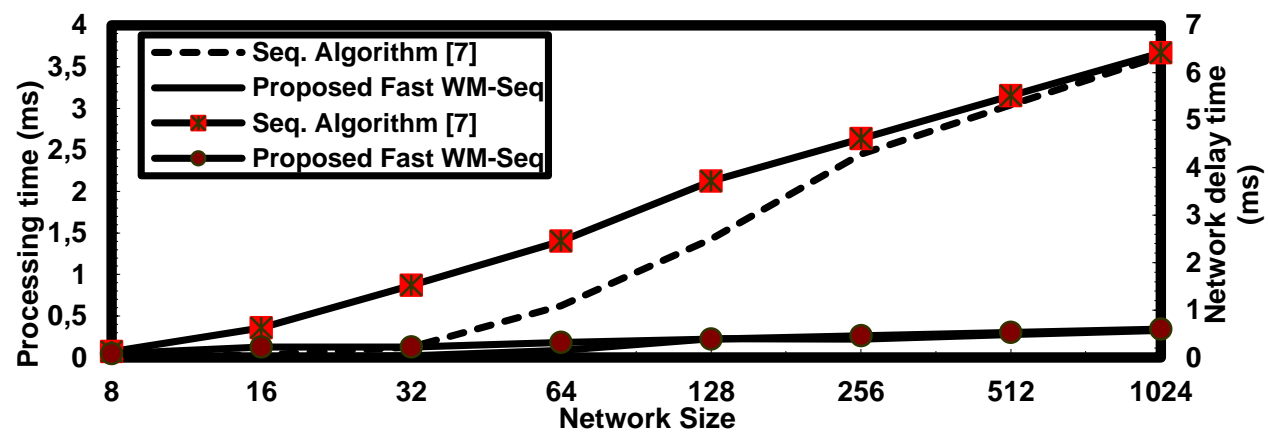

Figure 6. Processing time and network delay time variations against network size variations for the previous sequential algorithm and the proposed fast sequential window method

Figure 7 illustrates the relation between processing time and network delay time variations against the network size variations for the previous sequential down algorithm and the proposed fast sequential down window method. The processing time for the sequential down algorithm is $0.014 \mathrm{~ms}$, and the processing time for the proposed fast sequential down WM is $0.004 \mathrm{~ms}$ for the small network size value of 8 . The processing time for the sequential down algorithm is $0.68 \mathrm{~ms}$, and the processing time for the proposed fast sequential down WM is $0.154 \mathrm{~ms}$ for the medium network size value of 128 . The processing time for the sequential down algorithm is $1.256 \mathrm{~ms}$, and the processing time for the proposed fast sequential down WM is $0.3089 \mathrm{~ms}$ for the large network size value of 1024. In the same way, the network delay time for the sequential down algorithm is $0.031 \mathrm{~ms}$, and the network delay time for the proposed fast sequential down WM is $0.025 \mathrm{~ms}$ for the small network size value of 8 . The network delay time for the sequential down algorithm is $0.873 \mathrm{~ms}$, and the network delay time for the proposed fast sequential down WM is $0.303 \mathrm{~ms}$ for the medium network size value of 128. The network delay time for the sequential down algorithm is $1.58 \mathrm{~ms}$, and the processing time for the proposed fast sequential down WM is $0.507 \mathrm{~ms}$ for the large network size value of 1024 .

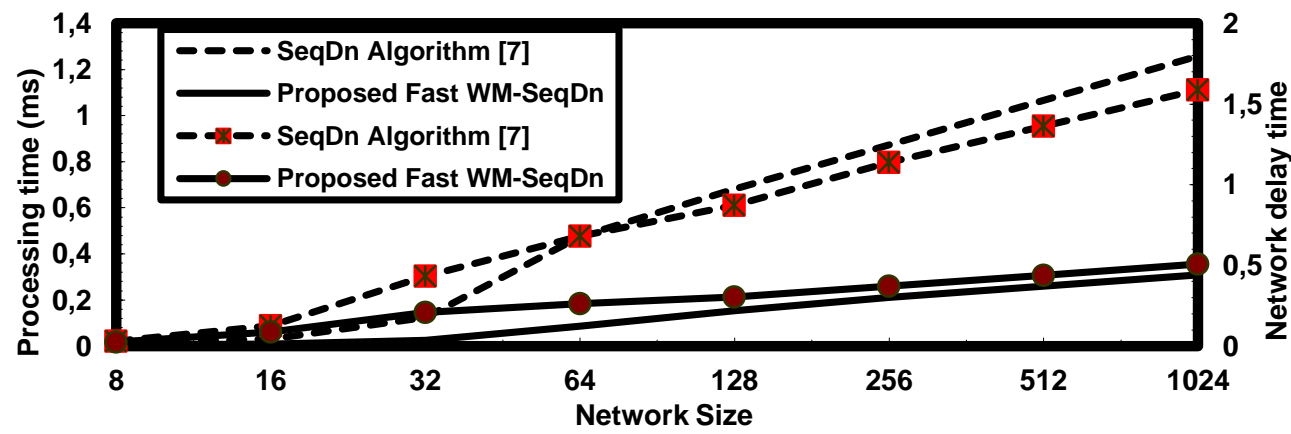

Figure 7. Processing time and network delay time variations against network size variations for the previous sequential down algorithm and the proposed fast sequential down window method 
The network blocking probability variations versus network size variations for various fast window methods and proposed routing algorithms is shown in Figure 8. The blocking probability for the WM is 0.0009 for the small network size value of 8 . The blocking probability for the WM is 0.0054 for the medium network size value of 128 . The blocking probability for the WM is 0.0098 for the large network size value of 1024. The blocking probability for improved WM is 0.0003 for the small network size value of 8 . The blocking probability for the improved WM is 0.0058 for the medium network size value of 128 . The blocking probability for the improved WM is 0.0987 for the large network size value of 1024 . The blocking probability for BWM is 0.0015 for the small network size value of 8 . The blocking probability for BWM is 0.254 for the medium network size value of 128 . The blocking probability for BWM is 0.3321 for the high network size value of 1024. The blocking probability for fast ascending WM is 0.0052 for the small network size value of 8 . The blocking probability for fast ascending WM is 0.217 for the medium network size value of 128 , while the blocking probability for fast ascending WM is 0.319 for the large network size value of 1024 . The blocking probability for fast descending WM is 0.004 for the small network size value of 8 , but the blocking probability for the fast descending WM is 0.145 for the medium network size value of 128. Meanwhile, the blocking probability for fast descending WM is 0.342 for thelarge network size value of 1024. The blocking probability for the fast sequential WM is 0.005 for the small network size value of 8 , but the blocking probability for fast sequential WM is 0.225 for the medium network size value of 128. Meanwhile, the blocking probability for fast sequential WM is 0.328 for the large network size value of 1024. The blocking probability for the fast sequential down WM is 0.0046 for the small network size value of 8 , but the blocking probability for fast sequential down WM is 0.1542 for the medium network size value of 128 . The blocking probability for the fast sequential down WM is 0.3089 for the large network size value of 1024 .

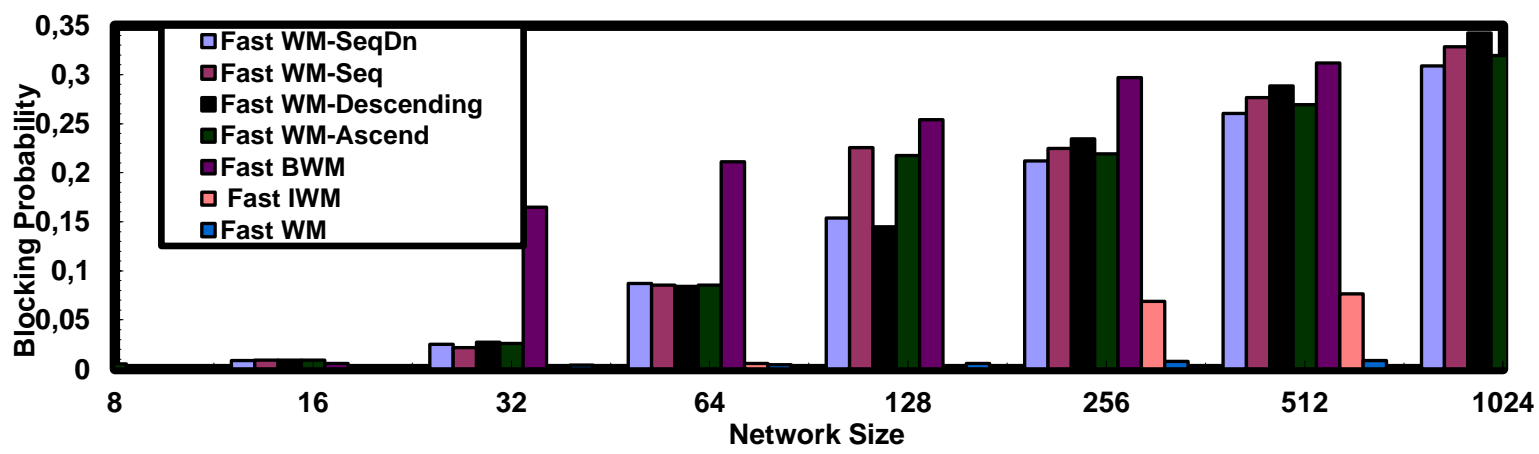

Figure 8. Blocking Probability variations versus network size variations for various fast window methods and the proposed routing algorithms

\section{CONCLUSION}

We have studied various routing algorithms and different fast window methods for upgrading the performance of optical computing networks. The processing time and network delay time variations against the network size variations for the previous routing algorithms and the proposed routing algorithms have been measured. In the same way, the Processing time and network delay time variations against the network size variations for the traditional window methods and the proposed fast window methods have been verified. The proposed fast window methods and routing algorithms presented a better network performance efficiency than the traditional window methods and the conventional routing algorithms. The fast window method presented the lowest network blocking probability compared to the other proposed window methods.

\section{REFERENCES}

[1] O. L. Ladouceur, "Breakthroughs in Photonics 2014: Optical Interconnection Networks" IEEE photonic journal, vol. 7, no. 3, pp. 751-756 June 2015.

[2] Manisha, and V. Malik, "Effect of Crosstalk in Optical Component", International Journal for Research in Applied Science \& Engineering Technology (IJRASET), vol. 3, no. 5, pp. 456-459, May 2015.

[3] R. R. Aggarwal et al., "Multistage Interconnection Networks: A transition from Electronic to Optical," Journal of Emerging Technologies in Web Intelligence, vol. 2, no. 2, pp. 142-147, May 2010.

[4] N. A. Yunus, and M. Othman "Reliability Evaluation and Routing Integration in Shuffle Exchange Omega Network", Journal of Networks, vol. 9, no. 7, pp. 1732-1737, Jul. 2014. 
[5] S. C. Chau et al., "Routing and Scheduling for a Novel Optical Multistage Interconnection Networks," Euro-Par 2005 Parallel Processing, Lecture Notes in Computer Science, vol. 3648, no. 9, pp. 984-993, 2005.

[6] C. Qiao, and R. Melhem, "A Time Domain Approach for Avoiding Crosstalk in Optical Blocking Multistage Interconnection Networks", Journal of Lightwave Technology, vol. 12. no. 10, pp. 1854- 1862, 1994.

[7] T. D. Shahida et al., "Fast Zerox algorithm for routing in optical Multistage interconnection networks", IIUM Engineering Journal, vol.11, no.1, pp. 28-39, 2010.

[8] A.K. Katangur et al., "Analyzing the performance of optical multistage interconnection networks with limited crosstalk", Cluster Computing, vol. 10, no.7, pp. 241-250, Mar. 2007.

[9] F. Abed and M. Othman, "Fast method to find conflicts in optical multistage interconnection networks," International Journal of The Computer Internet and Management, vol.16, no.1, pp. 18-25, 2008.

[10] M. Abdullah et al., "An efficient approach for message routing in optical omega network", International Journal of The Computer Internet and Management, vol.14, no.1, pp. 50- 60, 2006.

[11] M. A. Al-Shabi, "ZeroX Algorithms with Free crosstalk in Optical Multistage Interconnection Network" International Journal of Advanced Computer Science and Applications, vol. 4, no. 2, pp.156-160, 2013.

[12] IS Amiri et al., "Nonlinear Effects with Semiconductor Optical Amplifiers," Journal of Optical Communications, Vol. 0, Issue 0, Published Online: 12 April 2019, https://doi.org/10.1515/joc-2019-0053.

[13] Ramniwas, and L. Lodha "Reduce the Cross Talk in Omega Network by Using Windowing Techniques", International Journal on Recent and Innovation Trends in Computing and Communication, vol. 4, no. 6, pp. 54-57, Jun. 2016.

[14] U. Choudhury, and A. Sangiovanni-Vincentelli, "Constraint-Based Channel Routing for Analog and Mixed Analog/Digital Circuits," IEEE Transactions on Computer-Aided Design of Integrated Circuits and Systems, vol. 12, no. 4, pp. 497-510, April 1993.

[15] Ahmed Nabih Zaki Rashed et al., "The switching of optoelectronics to full optical computing operations based on nonlinear metamaterials," Results in Physics, Vol. 13, Article 102152, June 2019, https://doi.org/10.1016/j.rinp.2019.02.088.

[16] P. Saxena, and C.L. Liu, “A Post processing Algorithm for Crosstalk-Driven Wire Perturbation,” IEEE Transactions on Computer-Aided Design of Integrated Circuits and Systems, vol. 19, no. 6, pp. 691-702, June 2000.

[17] T. Gao, and C.L. Liu, "Minimum Crosstalk Channel Routing," IEEE Transactions on Computer-Aided Design, vol. 15, no. 5, pp. 465-474, May 1996.

[18] H. Zhou, and D.F. Wong, "An Optimal Algorithm for River Routing with Crosstalk Constraints," Proceedings of the International Conference on Computer-Aided Design, pp. 310-315, Nov. 1996.

[19] Ahmed Nabih Zaki Rashed et al., "Performance Evaluation of SAC-OCDMA System in Free Space Optics and Optical Fiber System Based on Different Types of Codes" Wireless Personal Communications Journal, Springer Publisher, Published online 17 May 2017, Vol. 96, No. 2, pp. 2843-2861, Sep. 2017.

[20] K. Jhang et al., "COP: A Crosstalk Optimizer for Gridded Channel Routing”, IEEE Transactions on ComputerAided Design of Integrated Circuits and Systems, vol. 15, no. 4, pp. 424-429, April 1996.

[21] M. Moudia, M. Othman, "Greedy Zero Algorithms for Conflict-Free Scheduling in Low Stage Interconnection Network," Procedia Computer Science, Vol. 59, pp. 106-114, 2015.

[22] G. Shanmugam, et al., "Meta heuristic algorithms for vehicle routing problem with stochastic demands," Journal of Computer Science, Vol. 7, pp. 533-542, 2011.

[23] M. Al-Shabi, M. Othman, "A new algorithm for routing and scheduling in optical omega network," International Journal of Advanced Computer Science and Applications, Vol. 16, No. 1, pp. 26-31, 2008.

[24] R. Bashirov R, T. Karanller, "On path dependent loss and switch crosstalk reduction in optical networks," Information Sciences, Vol. 180, No. 6, pp. 1040-1050, 2010.

[25] M. Moudi, M. Othman, "A Cost-Effective Architecture for Optical Multistage Interconnection Network," Journal of Networks, Vol. 8, No. 2, pp. 345-350, 2013.

[26] IS Amiri et al., "High-Speed Light Sources in High-Speed Optical Passive Local Area Communication Networks," Journal of Optical Communications, Vol. 0, Issue 0, Published Online: 20 April 2019, https://doi.org/10.1515/joc2019-0070.

[27] IS Amiri et al., "Spatial Continuous Wave Laser and Spatiotemporal VCSEL for High-Speed Long Haul Optical Wireless Communication Channels," Journal of Optical Communications, Vol. 0, Issue 0, Published Online: 24 April 2019, https://doi.org/10.1515/joc-2019-0061.

[28] IS Amiri et al., "Influence of Loading, Regeneration and Recalling Elements Processes on the System Behavior of All Optical Data Bus Line System Random Access Memory," Journal of Optical Communications, Vol. 0, Issue 0, Published Online: 15 August 2019, https://doi.org/10.1515/joc-2019-0163.

[29] IS Amiri et al., "The Engagement of Hybrid Ultra High Space Division Multiplexing with Maximum Time Division Multiplexing Techniques for High-Speed Single-Mode Fiber Cable Systems," Journal of Optical Communications, Vol. 0, Issue 0, Published Online: 19 Oct. 2019, https://doi.org/10.1515/joc-2019-0205.

[30] IS Amiri et al., "Performance Enhancement of Fiber Optic and Optical Wireless Communication Channels by Using Forward Error Correction Codes," Journal of Optical Communications, Vol. 0, Issue 0, Published Online: 24 Dec. 2019, https://doi.org/10.1515/joc-2019-0191.

[31] IS Amiri et al., "Comparative Simulation Study of Multi Stage Hybrid All Optical Fiber Amplifiers in Optical Communications," Journal of Optical Communications, Vol. 0, Issue 0, Published Online: 4 Feb. 2020, https://doi.org/10.1515/joc-2019-0132. 


\section{BIOGRAPHIES OF AUTHORS}
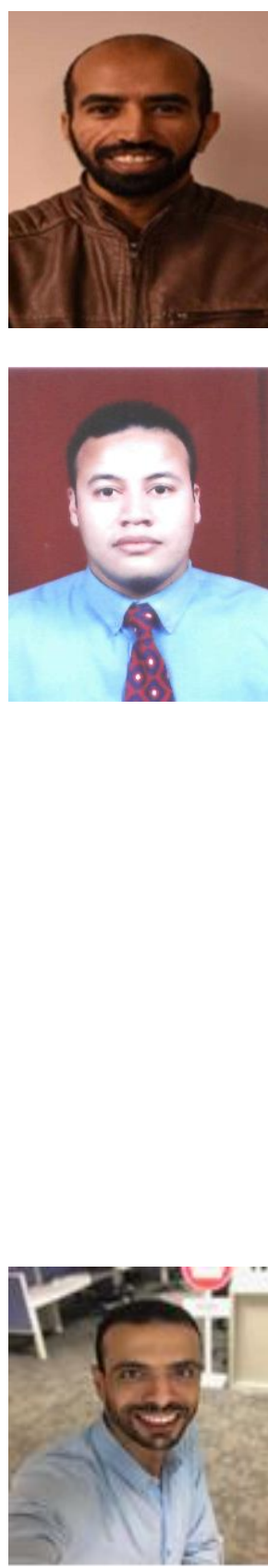

Assoc. Prof. Ahmed Nabih Zaki Rashed was born in Menouf city, Menoufia State, Egypt country in 23 July 1976. Received the B.Sc., M.Sc., and Ph.D. scientific degrees in the Electronics and Electrical Communications Engineering Department from Faculty of Electronic Engineering, Menoufia University in 1999, 2005, and 2010 respectively. Currently, his job carrier is a scientific lecturer in Electronics and Electrical Communications Engineering Department, Faculty of Electronic Engineering, Menoufia university, Menouf. Postal Menouf city code: 32951, EGYPT. His scientific master science thesis has focused on polymer fibers in optical access communication systems. Moreover, his scientific Ph. D. thesis has focused on recent applications in linear or nonlinear passive or active in optical networks. His interesting research mainly focuses on transmission capacity, a data rate product and long transmission distances of passive and active optical communication networks, wireless communication, radio over fiber communication systems, and optical network security and management. He has published more than 220 published scientific papers in international journals and conferences. He has published many high scientific research papers in high quality and technical international journals in the field of advanced communication systems, optoelectronic devices, and passive optical access communication networks. His areas of interest and experience in optical communication systems advanced optical communication networks, wireless optical access networks, analog communication systems, optical filters and Sensors. As well as he is editorial board member in high academic scientific International research Journals. Moreover, he is a reviewer member in high impact scientific research international journals in the field of electronics, electrical communication systems, optoelectronics, information technology and advanced optical communication systems and networks. His personal electronic mail ID (Email:ahmed_733@yahoo.com). His published paper under the title "High reliability optical interconnections for short range applications in high performance optical communication systems" in Optics and Laser Technology, Elsevier Publisher has achieved most popular download articles in 2013.

Eng. Ahmed M. El-Eraki was born in El-Zagazig, Al-Sharkya, Egypt in 12 March, 1988. Received the B.Sc. scientific degree in the Electronics and Communications Engineering Department from Faculty of engineering Sinai University, Egypt 2011. Master Degree in Electronics and Communications Engineering Department from Faculty of electrical engineering Menouf, Menofia University. Currently, his job carrier is Teacher Assistant in telecommunications Engineering Department, Faculty of Engineering, Egyptian Russian university, Badr city, Cairo Egypt.

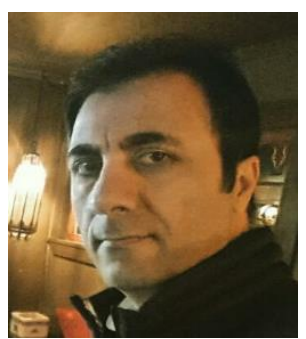

Dr. IS Amiri has been doing research on several topics such as the optical soliton communications, laser physics, fiber lasers, fiber grating, electro-optical modulators, nanofabrications, semiconductor design and modelling, Lumerical modelling, plasmonics photonics devices, nonlinear fiber optics, optoelectronics devices using 2D materials, semiconductor waveguide design and fabrications, photolithography fabrications, E Beam lithography, quantum cryptography and nanotechnology engineering. 